well as I remember, he followed the treatment as given for adults, although he did not use a large probe, I believe seldom one larger than No. 2 in a child.

DR. CASsius D. WESTcotT, Chicago-I saw two of these cases in the early years of my practice. I slit the canaliculus, passed a probe once, syringed the sac with boric solution, and after that simply expressed the contents of the sac until it was well. I happen to know that my old friend Dr. Holmes of Chicago has treated such cases suceessfully in this way, and he has seen a number of them.

Dr. C. W. HAwley, Chicago-From my own experience I should be inclined to think that it is not necessary to use probes, and the same would apply to the cutting of the canaliculus. A cut canaliculus will always be more or less trouble. some, for, in an infant I should imagine it would be almost impossible to make the typical incision of the canal.

\section{THE MADDOX ROD OR PHOROMETER: WHICH?*}

BY ALVIN A. HUBBEIL, M.D., Ph.D. BUFFALO, N. $\mathbf{Y}$.

The Maddox rod or suppressed-image or obscuration test in some of its forms, and the prism or diplopia test in some form of phorometer, are most commonly used in the determination of the co-ordination of the external muscles of the eyes. In this practice it is assumed that there is a functional position of rest for the eyes in binocular vision, and that in this position of rest, the lines of vision are both directed toward the point of fixation. It is not my purpose to discuss here the nature or limits of this co-ordination, the causes which disturb it, or the effects, or treatment of any disturbance of it. My only question is as to the comparative value of the diplopia test, by Stevens' phorometer.

Using Stevens' nomenclature, I assume that orthophoria is the strictly normal condition of ocular co-ordination, although I grant that there may be, within narrow limits and in relation to unusual accommodative or other forms of innervation, a condition of "physiological" heterophoria. I further assume that the object of co ordination tests is to determine the presence or absence of orthophoria, and if absent, the amount of variation.

During the whole period of my ophthalmic practice, I have used, more or less, the diplopia test, and for several years, as the most convenient form of it, the Stevens phorometer. Since Maddox brought forward the rod test, a number of years ago, I have also used this. and have made a large number of comparisons of the results of both.

Dissociation of the images of the two eyes is the principle governing each of these tests. In the diplopia test, the dissociation is effected by changing the visual axis of one eye by means of a prism. The displacement of one image can not be done without associating with it, more or less, an impulse to some form of ocular effort. This may be very slight in some cases and considerable in others. It may affect one muscle or set of muscles in some, and another in others. Certainly, there is not a state of complete muscular rest.

In the obstruction or suppression test, no such effort is invited, no change of innervation takes place. Binocular fixation is suspended without it, and the eyes yield to the deviation tendency in accordance with the status of innervation of the acting and governing nerve-centers. If this be true, it would seem that the Maddox rod should more correctly indicate the absence or presence and amount of heterophoria.

* Presented to the Section on Ophthalmology, at the Fiftieth Annual Meeting of the American Medical Association, held at Coumbus. Ohlo, June 6-9. 1999 .
COMPARATIVE REPORT OF 100 CASES, SHOWING 140 MEASURF. MENTS BY TIIE IFAUDOX ROD AND STEVENS' PIOROMETER. EIOPHORIA.
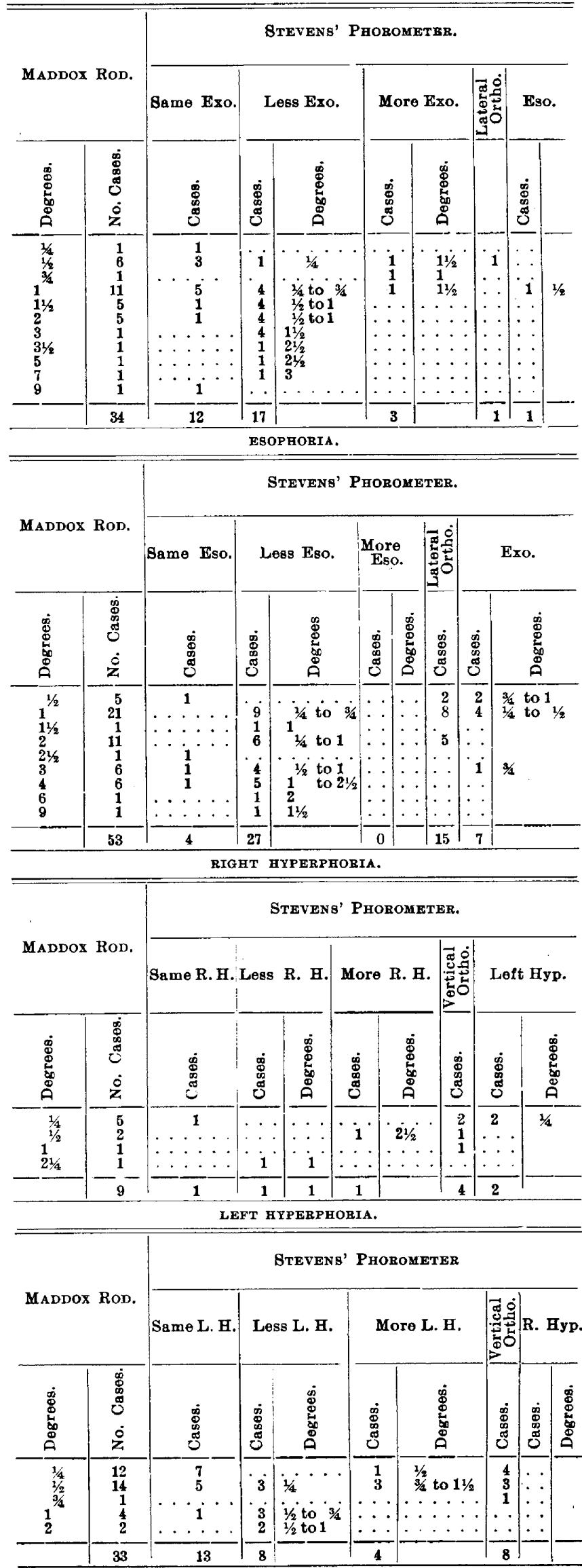
ORTHOPHORIA.

\begin{tabular}{|c|c|c|c|c|c|c|c|c|c|}
\hline \multirow{3}{*}{$\frac{\text { Maddox Rod. }}{\text { Cases. }}$} & \multicolumn{9}{|c|}{ Strevens' Phorometer. } \\
\hline & Ortho. & \multicolumn{2}{|c|}{ Eso. } & \multicolumn{2}{|c|}{ Exo. } & \multicolumn{2}{|c|}{ Right Hyp. } & \multicolumn{2}{|c|}{ Left Hyp. } \\
\hline & Cases. & 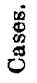 & $\stackrel{\dot{0}}{\circ}$ & $\begin{array}{l}\dot{8} \\
\text { \&. } \\
\text { की } \\
\dot{0}\end{array}$ & $\stackrel{\dot{0}}{\circ}$ & 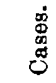 & $\stackrel{\dot{0}}{\circ}$ & 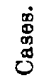 & $\dot{\otimes}$ \\
\hline $11 \ldots \ldots$ & 6 & . . & . . & 2 & $1 / 2$ & 1 & $1 / 2$ & 2 & $1 / 2$ \\
\hline
\end{tabular}

DEGREES SHOWN : TOTALS.

\begin{tabular}{|c|c|c|c|c|}
\hline & Eso. & Exo. & Rt. Hyp. & Left Hyp. \\
\hline $\begin{array}{l}\text { Btevens' Phorometer. . . . . } \\
\text { Maddox Rod. }\end{array}$ & $\begin{array}{l}393 / 4 \\
116\end{array}$ & $\begin{array}{l}443 / 4 \\
591 / 2\end{array}$ & $\begin{array}{l}43 / 4 \\
5 \% / 2\end{array}$ & $\begin{array}{l}201 / 4 \\
18 \% \\
\end{array}$ \\
\hline
\end{tabular}

Again, if it be contended that there is no extraneous muscular effort in the diplopia test, then, in the absence of such effort in either method, the one which, in general, shows the greater amount of heterophoria must be the most correct. Dr. Stevens and others hold that the Maddox rod does not reveal the full amount of heterophoria because of the tendency to fuse the images of the two eyes. In this case it would generally show less heterophoria than the phorometer, but the contrary has been my experience.

As an illustration of my experience, I will give the results of my measurements in 100 persons, taken consecutively as they came to my office, irrespective of conditions of general health or refraction, and without glasses. In this series I omitted cases in which vision was less than $5 / 12$ in either eye. The comparative tests were made at the same sitting, and at a distance of 5 meters, using a gas-flame about the size of that of a common candle.

In some of them, as will be inferred, the heterophoria was mixed, so to speak; that is, there was right or left hyperphoria with esophoria or exophoria.

In 53 cases in which the Maddox rod showed esophoria. Stevens' phorometer gave an esophoria of the same degree in 4, less esophoria and lateral orthophoria in 32 , exophoria in 7 , and more esophoria in none. In 34 cases in which there was exophoria by the rod, by the phorometer there was the same exophoria in 12 , less and lateral orthophoria in 18, esophoria in 1, and more exophoria in 3. In 9 cases of right hyperphoria by the rod, the phorometer showed the same in 1, less and vertical orthophoria in 5, left hyperphoria in 2, and more right hyperphoria in 1 . In 33 cases of left hyperphoria by the Maddox rod, the phorometer showed the same in 13 , less and vertical orthophoria in 16, right hyperophoria in none, and more left hyperphoria in 4 . In 11 orthophoric cases by the rod, the phorometer gave orthophoria in 6 , exophoria in 2 , right hyperphoria in 1 , and left hyperphoria in 2.

Thus it will be seen that in the 140 measurements the two tests gave the same results in 36 . In the heterophoric cases there was less heterophoria or, what was the same in effect, lateral and vertical orthophoria, by the phorometer than by the rod in $\% 1$, and in 8 there was more. The opposite form of heterophoria was shown in 10 , while in 7 of the orthophoric cases by the rod, there were exophoria and right and left hyperphoria by the phorometer. In view of the fact that by the rod test there is introduced, absolutely, no extraneous impulse to muscular contraction, and as its findings are in the large majority of cases equal to, or in excess of, those of the phorometer, I am forced to believe that, in connection with other contributory muscular tests, it is a more precise and trustworthy guide in daily practice than the phorometer.

The foregoing table shows more fully the comparative results of the 100 cases above referred to. DISCUSSION.

Dr. A. G. Blincoe, Bardstown, Ky.--Dr. Hubbell's conclusions are in the main correct. In my refraction work I have generally used the Stevens phorometer because I have considered it the simplest and most convenient method. I often find more difficulty in using the rod test. I have noticed, however, that after using the phorometer a number of times in the same case, there will sometimes be an appearance of orthophoria with it when the rod test will still show some heterophoria. It would thus appear that the rod test is the more aecurate of the two, but if in these cases the rod test had been used first, it might be that the phorometer would show want of equilibrium after orthophoria had been reached with the rod test, as the patient may soon learn to exercise some effort in fusing dissimilar as well as displaced images. We will all probably agree that it is generally better to use several tests and vary them occasionally or frequently.

In diagnosing and treating muscle errors we a! ways have to supplement these methods by the duction tests. In testing the adduction in a case recently, I found that with a prism base out before the right eye, the patient fused 25 degrees, while with the prism base before the left eye, he only fused 15 degrees. With the Stevens phorometer he showed 5 to 7 degrees of exophoria in one eye and orthophoria in the other, the exophoria appearing sometimes in one eye and sometimes in the other. With the Maddox rod before the right eye he had 2 degrees of exophoria, and before the left, 1.5 degrees. His abduction was about 15 degrees in each eye. It was a question with me to which eye the weak internal muscle belonged. The tropometer in experienced hands would probably have decided the matter.

By putting a red glass before the left eye while testing the adduction with a prism before the right in this case, the patient said the red image seemed to move toward and fuse with the other, and the same thing occurred in making the abduction tests.

In making some tests in my own person soon afterward, sitting twenty feet from a candle flame, before a dark background with a prism before one eye and a red glass before the other, I found the results both for abduction and adduction, in either eye, were the same. So it would appear from these tests that in making the right and left lateral duction tests, when a prism is held base in or out before the eye, we are testing the strength of the muscle of the other eye.

In another case of esophoria since then, the white light moved to the red with the weaker prisms, but when approaching near to the strongest fusing point, the red light seemed to move to the other one.

As these are points about which there is probably not a perfect unanimity of opinion in the profession, I should like very much to hear the views of any members present who have settled convictions in regard to them, and especially from any ono experienced in the use of the tropometer in such cases.

DR. W. L. PrLE, Philadelphia-When I first started to practice ophthalmology I used the Maddox rod. Later, I bought an optometer, which is really a Stevens phorometer, with two adjustable Risley prisms for duction measures, and on this I had the instrument-maker place a rotary Maddox rod. I made a number of tests with both phorometer and rod during a period of four months, and I concluded that the rod showed from 1 to 3 degrees of esophoria when the phorometer measured orthophoria. I feel perfectly satisfied with the use of the phorometer now, because $I$ believe the finding of the Maddox rod will always tend from I to 3 degrees toward the esophoric side, from the finding of the phorometer.

Dr. LUCIEN Howe, Buffalo, N. Y.-This subject lies rather near to my heart, and if you ask which of the two methods we shall trust implicitly I should say neither; that neither of these instruments will give the same readings in tests made at different times is due to the unconscious muscular effort that is made; this is usually accompanied by contraction of the pupil, and may be demonstrated by watching the constant action of the pupil as shown by the horizontal microscope. 
DR. G. C. Savage, Nashville, Tenn.-There is a reason for everything, whether we know that reason or not. It seems to me very clear that there is a field of binocular fixation, and if any part of the image falls on that field while making the muscle test there must necessarily be an error. The Maddox rod has come to stay, and it is going to do good service, but it will be especially in measuring the oblique muscles. So far as the older phorometers are concerned, the Stevens and the Wilson-and the latter is the better-neither one is built on the right principle. To do the work properly we should have a monocular phorometer, and I shall demonstrate one at the elose of this meeting. I think $I$ can show that it is more accurate than any phorometer heretofore.

Dr. I. V. WÜrdemann, Milwaukee, Wis.-I noticed in the series of cases given by Dr. Hubbell, that these eyes were all measured without the ametropia being corrected. In my opinion it is essential to correct this and make the eye emmetropic before an accurate estimate of the balance of the ocular muscles can be obtained, for the unreliability of our tests lies largely in the point brought out by Dr. Howe, the unconscious accommodation and convergence.

Dr. C. E. Nonton, Lewiston, Me.-I have found one great difficulty with the Stevens phorometer, which was to make the patient comprehend the situation and estimate the relative height of the two lights. After using it for some time I became convinced that it was not an accurate instrumert, for the results cbtained with it vary from time to time, to a considerable extent. I afterward combined a Maddox rod with a series of prisms, and found this a much more satisfactory instrument. Later I invented a more convenient instrument, consisting of a Maddox rod and a prism mobile; instruments essentially identical were invented by two ophthalmolegists and were placed on the market before I got mine done. I therefore purchased one of each kind and have used them for many years, and have found them much more satisfactory than the Stevens instrument.

Dis. A. A. IUubeell, Buffalo, N. X.-- I agree almost entirely with what Dr. Würdemann and Dr. Howe have said, with the exception of their reference to accommodation. I have not been able to make up my mind that the patient accommodated more in the rod test than in the diplopia test. $\mathrm{MI}_{\mathrm{y}}$ purpose has been simply to make a comparison between the Maddox rod and the phorometer, as to their immediate results, without any reference to treatment. Whether the eye was emmetropic or not, the conditions were the same during both examinations. I shall be very glad to hear more about Dr. Savage's phorometer.

\section{TREATMENT OF SEPTIC CONDITIONS IN CHILDREN.* \\ BY EDWIN ROSENTHAL, M.D. PHILADELPHIA.}

The presence of streptococcus pyogenes as a complication or sequel to many of the diseases of infancy or childhood exerts an influence on the course, duration or character of the disease so far as to give rise to new symptoms, utterly at variance with the initial malady. and significant of a dangerous, if not a fatal, result. As an independent affection the streptococci or the staphylococci are found so very frequently that they have given distinctive diseases.

Under the name "septicemia" is presented that complex disease which has for its origin the entrance of either of these microbes into the circulation, and by it is understood a poisoned blood. The term was originally employed by Koch for a condition of mierobic blood infection, in which the microbes multiply in the blood, and cause a rapidly fatal disease. The true name of this infection might be termed "strepto-infection" or "strepto-septicemia," or in the language of the bacteri-

* Presented to the Section on Diseases of Children, at the Fiftieth Annual Meeting of the American Medical Association, held at Columbus. Ohio, June 6-9, 1899. ologist, "streptomycosis." Whether the disease be a primary one, or whether it be a complication of, or a sequel to, another disease, this fact always remains: the strepto-infection is at first essentially a local disease; it is later that it becomes a blood infection.

The knowledge of its origin, thus shown, exerts an influence on the treatment, and it makes little difference whether the septic condition be a disease primarily, or a grave complication. The chief and certain rule is prophylaxis.

As a primary disease we may meet with septicemia in the new-born as well as in the older children, and as it is an infectious disease all the rules that govern such affections must be implied.

As the medical treatment, by which I mean the modern methods of combating sepsis, depends essentially on a true knowledge of the cause, it is necessary to make the diagnosis early and with the assistance of one who can make a bacteriologic test. As, however, it is not in the power of all to obtain a bacteriologic diagnosis, I will briefly summarize the clinical varieties of septic diseases that may be termed primary, whilst the same manifestations may hold good as a secondary sequel of the various infectious conditions.

The most frequent cases met with are in the newborn, and we meet them as follows:

Omphalitis: inflammation of the umbilicus, and cellulitis of the abdominal wall in the immediate neighborhood.

Inflammation of the umbilical vessels: in this condition, peritonitis, suppuration of the joints, erysipelas, multiple abscesses of the cellular tissue, sometimes suppurative parotitis, and atelectasis are common; pneumonia is also a common complication.

Peritonitis; this is the most frequent pathologic processes in pyemic conditions, and is often the cause of death.

Pneumonia: the most common form is the pleuropneumonia.

Pericarditis: rare and usually associated with pleurisy.

Endocarditis: this is very rare; Hirst reported a case.

Parotitis: I have seen this in an infant 4 days old; the mother was also so affected.

Meningitis: this is suggestive of injury during parturition.

Gastro-enteritis.

Pseudomembranous inflammation of the throat: this is not so rare, as instances have been reported by many; I have seen three cases, and in two I have seen membranous inflammation following on the operation of circumcision; in children 9 and 10 days old.

Osteomyelitis: when the septic condition has existed for some time, this may occur.

Joint suppuration: this is the same as in older people.

Abscess in the cellular tissue: this is the most frequently met with, and generally ends favorably.

Erysipelas: this, the cause of cellular abscesses, is met with in almost all cases of septic infection; not alone found in the umbilicus and neighboring tissues, but independently elsewhere, notably on the genitalia, simulating to a great extent syphilis congenitalis, and causing, in many instances, death by the swollen tissues pressing on the urethra and mechanically preventing urination, resulting in uremia and death. In the male I have seen circumcision a necessity, whilst in the female I have seen death resulting from the urinary infiltration following ulcerative action.

Septicemia as a secondary disease is most frequently 